\title{
Medical Student Research Day award winning abstracts
}

The Summer Research Program provides opportunities for first and second year medical students to participate in research projects being conducted by members of the Faculty of Medicine. Each year, the recipients of these studentships present their work at the Medical Student Research Day. This is an opportunity for students to present their own research and to be engaged by the work of their peers. Thirty-one medical students presented research projects in the form of oral or poster presentations this year. For the first time, the DMJ is proud to publish the award winning abstracts.

\section{Oral Presentation}

Nicholas Petropolis; Supervisor: Dr. Benjamin Rusak, Department of Psychiatry

\section{Diurnal Variation in Levels of Brain Neurochemicals}

Introduction: Proton magnetic resonance spectroscopy (1H-MRS) is a non-invasive method for brain imaging that is used to assess differences in levels of chemicals in the brain between psychiatric patients with various clinical conditions and controls, or between untreated and treated patients. Measuring such differences is made more difficult by the substantial test-retest variability of brain spectroscopic measurements. Question: Do daily fluctuations in brain metabolite concentrations contribute to this reported variability?

Hypothesis: A significant proportion of the normal biological variability in test-retest measures reported by previous studies might be accounted for by systematic physiological fluctuations associated with time of day.

Methods: Design: Participants were scanned every five hours throughout a twenty-five hour period on two onsecutive weekends. During the first weekend, subjects were allowed substantial nocturnal sleep (interrupted by a scan); during the following weekend they underwent complete overnight sleep deprivation. Participants: Three healthy males ages 24-25 with no history of neurological disease, psychiatric illness or sleep complaints. All participants scored as intermediate chronotypes on the Horne Ostberg morningness-eveningness questionnaire. Neuroimaging: 1.5 T GE Signa Scanner. H-MRS parameters: $\mathrm{TE}=35 \mathrm{~ms}, \mathrm{TR}=2000 \mathrm{~ms}, 320$ averages. Region of interest (ROI): $3375 \mathrm{~mm} 3$, in left prefrontal cortex. Four repeated, back-to-back, H-MRS acquisitions during each scan. Duration of scan approximately 1 hour. Processing of MRI and MRS Data: The percentage of gray and white matter within each ROI was calculated with the program AFNI. Metabolites were quantified using the program LCModel. Metabolite concentrations were normalized to percentage of white matter within each ROI.

Discussion: Carrying out this pilot study to completion was a significant achievement. We were able to show that it is possible to collect MRI data from multiple subjects in rapid succession in a limited time period, which is important for the feasibility of future studies. Assessing the waveforms for each metabolite in the presleep deprivation points, there is some apparent stability in the temporal patterns of metabolite concentrations across weeks, at least for $\mathrm{Cr}$, NAA and Glu/Gln. Comparing the range of concentrations within a time series with the following week, there is substantial overlap in all five metabolites. This overlap shows that there is some underlying stability in measurements and that the concentrations of at least some metabolites follow relatively stable daily patterns over successive weeks. In assessing the variation in metabolite concentration across 
time of day, two metabolites were found to have significant variations, as assessed by non-overlapping 95\% confidence intervals: Glu+Gln in week one, which rises from early to midday, and NAA in week 2 which has a trough in midday. This raises a red flag for other researchers who are studying Glu+Gln and NAA that they should be concerned about tracking circadian phase of scans.

\section{Poster Presentations}

Maria Currie; Supervisors: Dr. G.M. Hirsch, Dr. T.D.G. Lee, Department of Surgery

Allograft vasculopathy (AV) is the most common and refractory problem in late graft failure of heart transplants. The pathology of AV is well characterized; however, the inciting mechanism for this experiential pathology is contentious. There is no consensus on whether the intimal lesion formation is initiated by intimal endothelial cell damage or by medical smooth muscle cell (SMC) damage. Some research, predominantly with non transplant vascular inuury models, suggested that a threshold of medical SMC damage, and not intimal endothelial cell damage, determines the extent of intimal thickening during intimal lesion formation. The overall objective of this research is to determine the impact of endothelial versus medical damage in the progression of AV. The goal of this project was to develop a discriminatory transplant model that could be used to assess the impact of endothelial versus medical damage I the progression of AV. In order to evaluate alloresponses directed against the endothelium and the media in isolation, a discriminatory model was proposed involving the creation of chimeric aortic segments in which graft endothelium and media are of different strain origin. This would involve the isolation and de-endothelialization of mouse aortic grafts. These denuded grafts could then be transplanted into immunodeficient allogeneic recipients to re-endothelialize. The resulting chimeric grafts could then be re-transplanted into different recipients in which either the intima or the media would be seen as allogeneic. This would allow for the evaluation of whether damage to endothelium, or medial SMC, or both are required for lesion formation.

\section{Chris Jenkins; Supervisor: Dr. Bjorn} Nashan, Department of Surgery, Dalhousie University

\section{Characterization of T Regulatory Cells Generated by Oral Transplantation Tolerance}

Despite numerous advances in successful renal transplant, additional therapies are still warranted due to the numerous side effects or conventional immunosuppressants, as well as the inevitable failure of such medications to prevent chronic rejection events. Oral tolerance, the immune system ability to avoid mounting a destructive immune response against certain antigens encountered at mucosal surfaces, holds promise as a therapeutic tool in the future of allograft transplantation. We have previously demonstrated a role for $\mathrm{CD} 8+$ graft infiltrating cells (GICs) in maintaining oral tolerance to alloantigen in Lewis rats with Brown Norway rat renal allografts, however, little is known about the phenotype of these regulatory cells. We attempt to further characterize the CD8+ GICs from renal allografts of Lewis rats that had been fed Brown Norway rat splenocytes (experimental) or no alloantigen feeding (control) using flow cytometric staining for $\mathrm{a} / \mathrm{B}, \mathrm{y} / 8 \mathrm{~T}$ cells observed in experimental rat allograft GICs in comparison to control. Interestingly, adoptive transfer of total CD8+ splenocytes from experimental Lewis rats into a naïve Lewis rat was found to delay the rejection of a Brown Norway rat renal allograft. Intracellular flow cytometry staining for IL-4 did not reveal a significant percentage of IL-4 producing cells in either control or experimental animals, suggesting another method of detection should be evaluated. 